Ann. Zootech.; 1977, 26 (3), 429-434.

Note

\title{
Evolution de quelques paramètres physico-chimiques du contenu cæcal d'un poney au cours du nycthémère
}

\author{
J. L. TISSERAND, M. CANDAU (1), A. HOUISTE et C. MASSON \\ avec la collaboration technique de Catherine Coroelet et F. Faurie \\ Laboratoive de Recherches de la Chaive de Zootechnie de l'E.N.S.S.A.A., I.N.R.A., \\ 26, boulevard $D^{\mathrm{r}}$-Petitiean \\ a roI6 Dijon Cedex (France)
}

\begin{abstract}
Résumé
Sur un poney mâle, muni d'une canule permanente de cæcum, nous avons étudié l'évolution au cours du nycthémère du $\mathrm{pH}$ et des concentrations en azote total et ammoniacal ainsi qu'en acides gras volatils du contenu cæcal.

Par rapport à ce qui est connu pour le rumen des Polygastriques, les résultats obtenus montrent que, dans le cæcum du Poney, le $\mathrm{pH}(7,49)$ est plus élevé malgré une concentration moindre cn azote ammoniacal $(69 \mathrm{mg} / \mathrm{l})$. La teneur en acides gras volatils totaux (49 mmole/1) est plus faible avec une très forte proportion molaire d'acide acétique (69 p. 10o).

D'une manière générale, le contenu de cæcum paraît beaucoup moins variable au cours de la journée que celui du rumen.
\end{abstract}

\section{Introduction}

La possibilité pour le Cheval, herbivore monogastrique, d'utiliser les glucides membranaires grâce à une digestion microbienne dans son gros intestin est connue de longue date. Cependant peu de travaux ont été entrepris à ce jour pour étudier les fermentations microbiennes dans le cæcum et le côlon de cet animal (ARGENZIO, Southworth et Stevens i974, Hintz et al. i97ia, Hintz et Schryver I972, KERN et al. I973, Robinson et SlaDE 1974). Celles-ci n'ont pas l'importance de Cedex.

(1) Actuellement Chaire de Zootechnie E.N.S.A. de Toulouse, 145, avenue de Muret, 3 ro76 Toulouse 
celles constatées chez les Ruminants mais constituent néanmoins un facteur prédominant de l'utilisation digestive des aliments chez le Cheval.

Afin de compléter nos connaissances dans ce domaine, nous avons entrepris, depuis I972, au Laboratoire de Recherches de la Chaire de Zootechnie de l'École Nationale Supérieure des Sciences Agronomiques Appliquées (I.N.R.A.), une série d'études sur poneys munis de canule permanente du cæcum. Nous rapportons ici les premières observations effectuées dans le but de mieux connaître les variations de certains paramètres physicochimiques du contenu de cæcum au cours du nycthémère en les comparant en particulier à ceux du rumen du polygastrique.

\section{Matériel et méthodes}

\section{I. - Matériel}

Un poney mâle entier, âgé de sept ans, pesant ${ }_{5} 5^{\circ} \mathrm{kg}$, muni d'une canule permanente du cæcum, reçoit deux repas par jour (à $8 \mathrm{~h}$ et $\mathrm{I} 7 \mathrm{~h}$ ) composés de foin de pré à volonté et de $0,5 \mathrm{~kg}$ d'avoine mis à la disposition de l'animal une heure après le début du repas de foin.

L'eau est laissée à la disposition de l'animal en permanence.

\section{2. - Méthodes}

a) Technique de fistulation.

La canule cæcale est mise en place sous anesthésie générale par le penthiobarbital sodique (Nesdonal) après une prémédication avec un tranquillisant. L'intervention commence par une incision cutanée puis musculaire sur une dizaine de centimètres au niveau du creux du flanc droit de l'animal.

Après avoir isolé le péritoine, le cæecum est extériorisé pour la mise en place immédiate de la canule (tube de chlorure de polyvinyle). Pour une meilleure cicatrisation, le segment libre de la canule est placé, grâce à une étroite boutonnière, sur le côté aval de l'incision. Une antibiothérapie post-opératoire est effectuée pendant 4 jours.

\section{b) Technique de prélèvement.}

Des prélèvements de Ioo $\mathrm{ml}$ de contenu cæcal sont réalisés à l'aide d'une sonde reliée à une pompe à vide toutes les heures pendant 24 heures et ceci est répété 5 fois à raison d'une journée par semaine.

c) Mesures.

Sur chaque prélèvement sont effectuées les mesures suivantes :

- mesure du $\mathrm{pH}$

- dosage de l'azote total (Kjeldahl)

- dosage de l'azote ammoniacal $\mathrm{N}^{-\mathrm{NH}_{3}}$ (méthode Conway) racétique)

- dosage de l'azote protéique (obtenu par précipitation à l'acide trichlo-

- dosage des acides gras volatils : AGV (par chromatographie en phase gazeuse : colonnes de $3 \mathrm{~m}$ long, remplies de chromosorb W. $80-$ I00 $\mathrm{m}+\mathrm{NPGA}$ 25 p. Ioo et acide orthophosphorique 2 p. Ioo). 
TABLEAU I

Teneurs moyennes en azote et en acides gras volatils totaux du contenu de cocum du poney Average nitrogen and total volatile fatty acids concentration in the caecum of the pony

\begin{tabular}{|c|c|c|c|c|c|c|c|c|}
\hline $\begin{array}{l}\text { Données } \\
\text { (Data) }\end{array}$ & $\mathrm{pH}$ & $\begin{array}{l}\text { Azote } \\
\text { total } \\
(\mathrm{mg} / \mathrm{l})\end{array}$ & $\begin{array}{l}\text { Pro- } \\
\text { téines } \\
\text { (mg / } / \mathrm{l})\end{array}$ & $\begin{array}{c}\text { Azote } \\
\text { ammo- } \\
\text { niacal } \\
(\mathrm{mg} / \mathrm{l})\end{array}$ & $\begin{array}{c}\text { Acides } \\
\text { grass } \\
\text { volatils } \\
\text { (mmole } / 1 \text { ) }\end{array}$ & $\begin{array}{c}\text { Acétique } \\
\text { (p. Iоo) }\end{array}$ & $\begin{array}{l}\text { Propio- } \\
\text { nique } \\
\text { (p. 10o) }\end{array}$ & $\begin{array}{l}\text { Buty- } \\
\text { rique } \\
\text { (p. 100) }\end{array}$ \\
\hline $\begin{array}{c}\text { Valeur } \\
\text { moyenne } \\
\text { (Meant value) }\end{array}$ & 7,49 & .584 & 302 & 69 & 49 & 69 & 23 & 5.3 \\
\hline $\mathrm{S} x$ & 0,13 & L 80 & $\therefore \quad 43$ & \pm 22 & $\therefore \quad 9$ & 4 & $\vdots$ & $\therefore 0,7$ \\
\hline
\end{tabular}
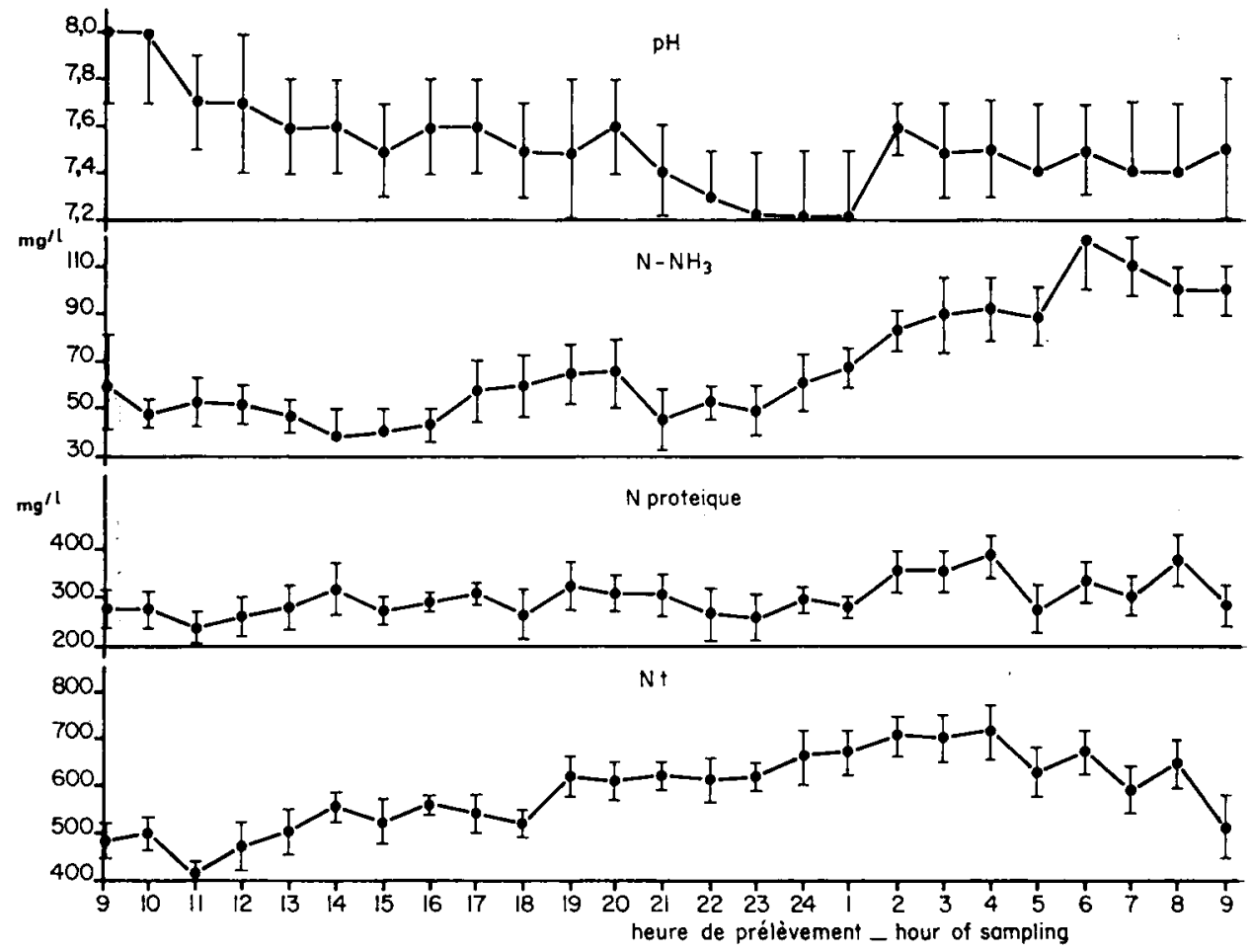

1.1. 1. - Variations $d u p H$ et des concentrations en azote ammoniacal, asote protéique et azote total dans le contenu de cacum de poney au cours de la journée. $(!-\longrightarrow$ - écart-type de la moyenne)

pH values and $\mathrm{N}-\mathrm{NH}_{3}$, protein $\mathrm{N}$ and total $\mathrm{N}$ concentrations in pony cacum during the day.

(|-- - staudard error of the mean). 


\section{Résultats - Discussion}

Les résultats moyens obtenus au cours des cinq séries de mesures sont résumés dans le tableau I. Il montre que :

Le $\mathrm{pH}$ du contenu de cæcum, pour un régime composé de foin et de céréales, est basique et reste compris entre 7 et 8 . Malgré quelques écarts $(0,2$ à 0,3 pts) entre les mesures à une même heure, il reste relativement stable au cours du nycthémère.

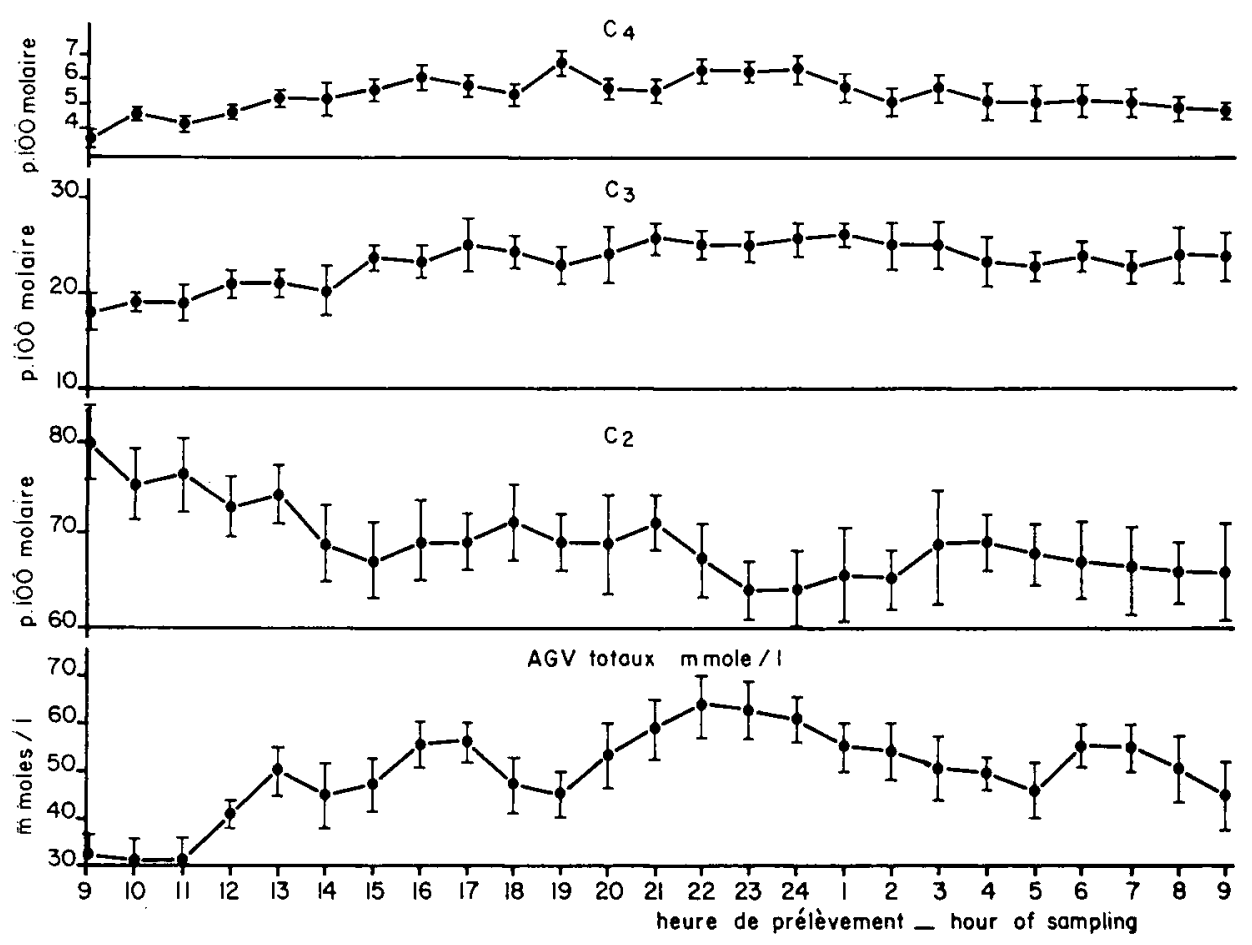

FIG. 2. - Variations de la teneur en acides gras volatils totatx dans le contenu de cacum de poney et des pourcentages molaires on $C_{2}, C_{3}, C_{4}$ an cours de la journée (1-- écart-type de la moyenne).

Total volatile fatty acids concentration in the caecum of the pony and acetic, propionic, butyric molar percentages during the day

(- - standard error of the mean).

L'azote du contenu de cæcum est composé principalement de protéines 52 p. IOo) et son taux varie peu au cours de la période de 24 heures.

La teneur en azote ammoniacal $\left(\mathrm{N}-\mathrm{NH}_{3}\right)$ reste faible (I2 p. Ioo de l'azote total) mais présente des variations au cours de la journée. Il en est de même de la production d'acides gras volatils (AGV) (50 millimoles par litre) qui sont représentés essentiellement par l'acide acétique (70 p. Ioo).

Par rapport à ce qui se passe dans le rumen du polygastrique, nous constatons une valeur du $\mathrm{pH}$ beaucoup plus élevée dans le cæcum du poney bien que la production d'N-NH $\mathrm{H}_{3}$ soit beaucoup plus faible. 
La production d'acides gras volatils, pour sa part, est moins importante dans le cæcum du poney que dans le rumen. L'acide acétique y est prédominant, ce qui a déjà été noté par ULYATT et al. I975 dans le cæcum de ruminant.

Les graphiques I et 2 , qui représentent les variations enregistrées au cours du nycthémère, indiquent que la stabilité du contenu du cæcum est beaucoup plus grande que celle du rumen. Une évolution est enregistrée pour la concentration en $\mathrm{AGV}$ et en $\mathrm{N}-\mathrm{NH}_{3}$. Le pH, pour sa part, est peu modifié. Il présente toutefois deux périodes de baisse : la première entre Io h et $\mathrm{I} 4 \mathrm{~h}$ et surtout la seconde entre $20 \mathrm{~h}$ et $24 \mathrm{~h}$. L'acidification constatée est imputable à une augmentation de la production d'AGV et surtout à un accroissement des taux d'acides propionique et butyrique durant ces deux périodes.

Par contre, la formation d'N-NH semble décalée dans le temps puisque l'augmentation de la concentration en cet élément se situe entre $16 \mathrm{~h}$ et $20 \mathrm{~h}$ et surtout entre o h et $6 \mathrm{~h}$. Ce dernier phénomène, difficilement explicable, pourrait être dû au fait que l'azote ammoniacal provenant surtout de protéines membranaires ne serait formé et libéré qu'après l'attaque des membranes et notamment la digestion des glucides solubles et de la cellulose. Par contre, l'évolution du $\mathrm{pH}$ et des AGV nous incite à penser que les périodes de Io h à $\mathrm{I} 4 \mathrm{~h}$ et surtout de $20 \mathrm{~h}$ à $24 \mathrm{~h}$ sont des périodes d'activité intense du cæcum par suite de l'arrivée vraisemblable de contenus digestifs consécutifs au repas : $8 \mathrm{~h}$ et $\mathrm{I} 7 \mathrm{~h}$.

Ces premières observations sont en accord avec les principaux résultats déjà publiés (Hintz et al. I97Ib, Kolb et GürtLER I97I, CANDAU et Vigroux I974, Robinson et Slade I974, Tisserand I975).

Bien que les valeurs obtenues ne soient que la résultante d'activités différentes telles que : digestion microbienne, absorption des nutriments, rythme de remplissage et de vidange du cæcum, nos résultats permettent de penser que les variations de composition du contenu sont beaucoup moins importantes dans le cæcum du cheval que dans le rumen. Ceci est sans doute imputable au fait que le cæcum ne reçoit que les résidus de la digestion chimique des aliments dans l'intestin grêle (RUCKebl'sch, Vigroux, Candau, I976).

Accepté pour publication en mars 1977.

\section{Summary}

\section{Variation of some physico-chemical parameters in the cacum content of a pony during the $24 \mathrm{hr}-\mathrm{cycle}$}

A pony fitted with a caecum fistula was used to study the variation of $\mathrm{pH}$ and $\mathrm{N}-\mathrm{NH}_{3}$, protein- $\mathrm{N}$, total- $\mathrm{N}$ and total volatile fatty acid concentrations during the $24 \mathrm{hr}$-cycle.

In comparison with results generally obtained on the polygastric rumen, the $\mathrm{pH}$ of the pony caecum (7.49) was higher in spite of a smaller $\mathrm{N}-\mathrm{NH}_{3}$ concentration (69 $\left.\mathrm{mg} / 1\right)$. The total volatile fatty acid concentration was lower $(49$ mmole /1), but the molar percentage of acetic acid (69\%) was very high.

According to the results obtained, the caecum content scems to be less variable than the rumen content during the 24 hr-cycle.

\section{Références bibliographiques}

Argenzio R. A., Southworth M. et Stevens C. E., 1974. Sites of organic acid production and absorption in the equine gastro-intestinal tract. Am. J. Phys., 226, 1043-1050.

Candau M., Vigroux Ph., I974. Mécanisme de l'hypermotilité cacale liée au repas chez le cheval. C. R. Soc. Biol, 168, 893-899. 
Hintz H. F., Hogue D. E., Walker E. F. (Jr.), Lowe J. F., Schryver H. F., i97ia. Apparent digestion in various segments of digestion tract of ponies fed diets with varying roughage grain ratios. J. Anim. Sci, 32, 245-248.

Hintz H. F., Argenzio R. A. et Schryver H. F., 197Ib. Digestion coefficients blood glucose levels and molar percentage of volatil acids in intestinal fluid of ponies fed varying forage grain ratios. J. Anim. Sci., 33, 992-995.

Hrntz H. F., Schryver H. F., I972. Nitrogen utilisation in ponies. J. Anim. Sci., 34, 592-595.

Kern D. L., Slyter L. L., Weaver J. M., Leffel E. C., Samuelson G., I973. Pony caecum VS steer rumen, the effect of oats and hay on the microbial ecosystems. J. Anim. Sci., 37, 463469.

Kolb E. et GürTLER M., 197I. Ernahhungsphysiologie der Landwirtschaftlichen Nutztieve. G. Fisher Verlag, Jena, 309-316.

Robinson D. W., SLAde L. M., 1974. The current status of knowledge on the nutrition of equine. J. Anim. Sci., 39, 1045-1049.

Ruckebusch Y., Vigroux Ph., Candau M., 1976. Analyse du comportement alimentaire chez les équidés. Joumées d'étude des recherches sur les équidés (Paris Io mars 1976). Journées du C.E.R.E.O.P.A.

Tisserand J. L., I975. Contribution à l'étude de la digestion dans le cæcum du cheval. Journées d'étude des recherches sur les équidés. (Paris 5 mars 1975). Journées du C.E.R.E.O.P.A.

Ulyatt M. J., Dellow D. W., Reid C. S. W., Bauchop T,, I975. Structure and function of the large intestine of ruminants. Proc. IVth International Symposium of Ruminant Physiology, Sydney Australia. August 1974, I 19-133. 